\title{
Induced pluripotent stem cells in psychiatry: a critical review
}

Alejandro De Los Angeles ${ }^{1,2}$, Kristen J Brennand ${ }^{3-5}$, Nicola A. Hall ${ }^{1,2}$, Michael B Fernando ${ }^{4,6}$, Paul J Harrison ${ }^{1,2}$, Brady J Maher-8, Daniel R Weinberger ${ }^{6-11}$, Elizabeth M Tunbridge ${ }^{1,2}$

1. Department of Psychiatry, University of Oxford, Oxford, UK

2. Oxford Health NHS Foundation Trust, Oxford, UK

3. Department of Genetics and Genomic Sciences, Icahn School of Medicine at Mount Sinai, New York NY, US

4. Nash Family Department of Neuroscience, Icahn School of Medicine at Mount Sinai, New York NY, US

5. Department of Psychiatry, Icahn School of Medicine at Mount Sinai, New York NY, US

6. Graduate School of Biomedical Science, Icahn School of Medicine at Mount Sinai, New York, NY, USA

7. Lieber Institute for Brain Development, US

8. Department of Psychiatry, Johns Hopkins University School of Medicine, Baltimore, MD, USA

9. The Solomon H Snyder Department of Neuroscience, Johns Hopkins School of Medicine, Baltimore, MD, USA

10. Department of Neurology, Johns Hopkins School of Medicine, Baltimore, MD, USA

11. Department of Genetic Medicine, Johns Hopkins School of Medicine, Baltimore, MD, USA

Running title: iPSCs in psychiatry

Corresponding author: Elizabeth Tunbridge

University Department of Psychiatry

Warneford Hospital

Oxford, OX3 7JX, UK

$+44(0) 1865618328$

Elizabeth.tunbridge@psych.ox.ac.uk

Key words: schizophrenia, bipolar disorder, model system, neuron, pathophysiology, drug screening 


\begin{abstract}
:
A key challenge in psychiatry research is the development of high-fidelity model systems that can be experimentally manipulated to explore and test pathophysiological mechanisms of illness. In this respect, the emerging capacity to derive neural cells and circuits from human induced pluripotent stem cells (iPSCs) has generated significant excitement. This review aims to provide a critical appraisal of the potential for iPSCs in illuminating pathophysiological mechanisms and to situate them in the wider technical landscape. We discuss the selection of iPSC phenotypes relevant to psychiatry, the information that researchers can draw on to help guide these decisions, and how researchers choose between the use of 2D cultures, vs. more complex 3D model systems. We discuss the strengths and limitations of current models, and the challenges and opportunities that they present. Finally, we discuss the potential of iPSC-based model systems for clarifying the mechanisms underlying genetic risk for psychiatry, and the steps that will be needed to ensure that robust and reliable conclusions can be drawn. We argue that, whilst iPSC-based models are ideally placed to study fundamental processes occurring within and between neural cells, they are often less well-suited for case-control studies, given issues relating to statistical power and the challenges in identifying which cellular phenotypes are meaningful at the level of the whole individual. Our aim is to highlight the importance of considering the hypotheses of a given study to guide decisions about which, if any, iPSC-based system is most appropriate to address it.
\end{abstract}


Psychiatric disorders are challenging to study mechanistically as they are human-specific and result from the complex dysfunction of a largely inaccessible tissue. Accordingly, putative brain cells derived from human induced pluripotent stem cells (iPSCs), which have genomes of real people and can be manipulated experimentally, have been enthusiastically adopted as potential model systems. Many recent reviews cover their possible advantages and applications, both clinically and as research tools $(1,2)$. However, few have critically appraised their use and attempted to situate them in the wider technical and conceptual landscape of psychiatric research (Figure 1). Therefore, we attempt to do so here. We also provide some guidance for the design and reporting of iPSC studies in psychiatry (Table 1).

iPSC systems are extremely well suited to experiments investigating the impact of manipulations on fundamental molecular and cellular processes and, potentially, on aspects of neurodevelopment. However, their utility in other situations is substantially less clear-cut. This is particularly the case for studies that seek to link cellular measures with psychiatry-relevant phenotypes observed at the level of the whole individual which, by their nature, are typically complex and temporally dynamic. Some of these challenges are purely logistical - relating to sample size and statistical power, for example - but others are more conceptual in nature; for instance: even if robust relationships are observed between phenotypes at the cellular and whole-individual levels, how do we determine which (if any) of these are meaningful. We recommend that both nascent and established iPSC researchers return to the hypothesis that they seek to test and ask themselves these fundamental questions: what is it you want to model, and is the proposed iPSC platform able to achieve this with high fidelity? We believe that reflecting on these key questions is critical for determining whether iPSC-based systems can help disentangle that which is meaningful from that which is merely possible.

iPSCs in psychiatry: a (very) brief history

Initial studies employing iPSCs in psychiatry focused primarily on monogenic conditions. iPSCderived neurons from a small sample of individuals with Rett syndrome showed reduced dendritic spines and synapses, and altered electrophysiological properties, compared with controls (3). The first study to use iPSC-derived neurons to explore functional changes in a polygenic psychiatric disorder - schizophrenia - also reported evidence for decreased neuronal connectivity in four patient-derived samples, compared with four controls, but in the absence of altered electrophysiological parameters (4). Researchers have also used patient-derived iPSC neurons to explore the potential cellular correlates of medication response (5). However, all these studies were conducted in extremely small samples (group sizes of 3 - 6) using a between-subjects approach, raising concerns about statistical robustness and generalizability. Therefore, more recent studies have moved towards investigations using isogenic controls to control for inter-individual differences in genetic background (6-9). Nevertheless, variable methodological approaches are used across the field and, in most cases, there is little or no evidence for replication of observed findings.

Early studies all utilized 2D cultures. A more recent development is the establishment of 3D cultures, termed brain organoids or spheroids. A few studies have compared 3D models derived from patients (with autism (10), schizophrenia (11) or bipolar disorder (12)) vs. controls. Like the approaches employed in 2D cultures, researchers are beginning to use organoids to explore the impact of genes harboring rare, penetrant disorder-associated mutations on neurogenesis and gene expression $(13,14)$. Given their appeal as a potential window into human neurodevelopment and the early stages of psychiatric disorder pathology, it is likely that studies in organoids will become more widespread. However, most psychiatric organoid experiments remain small in scale and so essentially represent proof-of-principle studies. 
Psychiatric illnesses in a dish? The questions of phenotype and cell type

The potential of iPSC models is to approximate biological aspects of illness or risk in the context of a particular human cell, with a particular human genome, which may then be studied to illuminate pathogenic mechanisms and to identify targets for remediation or rescue. Arguably the biggest challenge in realizing this potential is to identify the most appropriate phenotype(s) to measures and the most suitable cell types to study. How straightforward this is will depend on the hypothesis, as well as practical and technical constraints. For studies focused on specific genes, the phenotypic readout may be relatively clear, at least theoretically. For example, if the goal is to examine the effect of (rare) disease-associated coding mutations in an ion channel gene, it makes sense to determine the biophysical and electrophysiological properties of the resulting channels (15), and the function of downstream effector pathways. Similarly, iPSCbased models are extremely well-suited for the study of fundamental cellular processes, particularly those which require cells to have a neural identity, such as dendritic outgrowth and synapse formation. However, it is often less obvious what the appropriate assay should be, particularly in cases where the molecular target of interest is of unknown function; this can be the case even for well-studied genes if they give rise to multiple, functionally distinct protein products $(16,17)$ or are involved in a variety of molecular pathways.

Decisions about phenotype(s) are particularly challenging for case-control studies, as the absence of any pathognomonic neuropathological changes in patients with psychiatric disorders (in contrast to neurodegenerative diseases) means that there is no clear - let alone diagnostically significant - disease-associated phenotype to model at the cellular level. Conversely, we do not know whether a phenotype in a rarefied cell model of early neuronal development would be expected to mirror characteristics of illness in mature brains, even if the cell model phenotype reflects a basic pathogenic mechanism. Several studies have demonstrated changes in dendritic outgrowth and synaptic density in patient-derived iPSC neuronal cultures $(4,18)$, mirroring at least superficially evidence for alterations in neuropil and synaptic density reported in adults with some psychiatric conditions, particularly schizophrenia (19-21). However, other case-control phenotypic differences are less clearly allied to neuropathological observations. For example, it is hard to square the substantial reductions in neuronal viability observed in cultures derived from iPSCs from patients with bipolar disorder (22) with the limited evidence for changes in neuronal number in the brains of patients (23). Similarly, findings of disorganized migration of neural progenitor cells in brain organoids derived from patients with schizophrenia (11) are inconsistent with the lack of evidence for neuronal disarray in postmortem brain tissue $(21,24)$. Clearly, it is possible that some of the case-control differences observed in iPSC systems occur transiently in the disease state during development. However, they may be inaccurate renderings of genuine group differences, owing to differences between iPSC models and the in vivo environment. For example, differences in neurotransmitter function in the mature brain may manifest as changes in migration in vitro, since many neurotransmitters act as trophic factors during development (25), whilst the lack of microglia, and concomitant reduction in synaptic pruning (26), in many iPSC models may confound phenotypes relating to synaptic size and/or number. Finally, group differences may also represent false leads, as the result of insufficient sample size or related to the vagaries of the in vitro environment (27), for example, and therefore should be treated with caution.

There has been a tendency in reports to date to try to squeeze findings into loosely linked clinical phenomena. For example, alterations in catecholamine release have been reported in iPSC-derived dopamine neurons derived from schizophrenia patients, compared with controls (28), and have been taken as evidence for consistency between in vivo and in vitro measures (29), arguing that in schizophrenia there is excessive DA release in striatum. However, changes in dopamine synthesis fluctuate within individuals over time (30), and track the phase of the 
illness (31), suggesting that it is a marker that is (at least in part) 'state' rather than 'trait'. Although there is extensive wiping of epigenetic (and therefore presumably 'state') marks during reprogramming, it may be incomplete in some circumstances $(32,33)$, including at least one locus of relevance to psychiatry (the Fragile X locus, FMR1)(3). It will therefore be crucial to assess whether and how these residual signatures are related to 'state' markers, including fluctuations in the psychiatric symptoms of the donor. This understanding will be critical if we are to understand how (and if) iPSC models, taken at a relatively few moments in the changing developmental landscape of early cell differentiation, can predict anything meaningful about the aspects of the illness in those from whom the cells were taken.

A major obstacle to the accurate modeling of neuropsychiatric disorders in vitro is the relative immaturity of the derived neural cells. Gene expression and electrophysiological studies indicate that cells within both 2D and 3D models remain broadly similar to early fetal neurons (34-36). Notably, although often cited as a limitation, the fetal-like identity of iPSC-derived neural cells may have some advantages, at least in theory. If they truly recapitulate aspects of human neurodevelopment (discussed further below), they may provide a window into processes that are otherwise largely inaccessible. Therefore, for researchers focused on psychiatric disorders with a neurodevelopmental origin, such as schizophrenia (37) and autism (38), iPSC-based models may provide a system in which to study cause and effect relationships.

One of the appealing aspects of iPSC-based models of psychiatric disorders is their potential to identify cellular phenotypes that might predict information about an individual's illness course or medication response. Accordingly, there is much interest in using such phenotypes as a functional readout for high-throughput drug screening to identify new therapeutic approaches in a target-agnostic manner ('phenotypic drug discovery')(39). For this potential to be realized, it will be important to identify phenotypes that are relevant to the illness experienced by those from whom the cells were taken and/or which reliably predict clinical improvement. To date, the best-studied area is lithium responsiveness in patients with bipolar disorder, although examples also exist for other psychiatric disorders, e.g. autism (40). Mertens and colleagues (5) demonstrated hyperactivity in iPSC-derived dentate gyrus neurons derived from patients, which was reversed by lithium only in those derived from patients who were lithium responsive. In follow-up studies, the same group replicated these findings, successfully predicted the lithium responsiveness of a patient using a classifier trained on electrophysiological parameters of the responder vs. non-responder cells (41), and suggested that hippocampal CA3 hyperexcitability might be unique to lithium responders (42), and reduced by lithium exposure (43). However, the studies conducted to date have been used small samples and have emerged from a single laboratory. As promising as this appears, it will be important to ensure that this phenotype is reliable in a larger sample and, crucially, that it is robust across laboratories.

In conclusion, since there is no single cellular phenotype relevant to all situations in psychiatry or even for any specific disorder - the decision as to what to study in iPSC-based systems is unclear. Indeed, we would argue that approaching studies from a standpoint of 'what to study?' is fundamentally misguided in many cases. Arguably, the relevant question in most situations is not "what phenotype is best placed to identify group differences?" but rather "what phenotypic read-out is best suited to provide meaningful information about my manipulation/comparison of interest?" An examination of the literature suggests that group differences are relatively easy to detect at the cellular level - we are unaware of any negative study in psychiatry - but the importance of this observation remains opaque. Assuming it is not the result of publication bias, it is possible that iPSC-based systems, freed from the selection pressures and compensatory mechanisms that operate at the level of larger-scale neural systems and the whole organism, are uniquely placed to identify relationships between cellular phenotypes and manipulations of 
interest. However, it remains a significant challenge to determine which of these relationships is meaningful in terms of pathophysiology or prognosis. Ultimately, the onus is on individual investigators to be fully transparent about their motivations and how this relates to their findings. Hypothesis-free studies can be extremely valuable, not least in terms of providing a resource for the community (44-48) and, potentially, for identifying predictors of patient symptoms or outcome. Nevertheless, post-hoc explanations retrofitted to cellular observations are of much less value, and in the long run have the potential to damage the reputation of the very model systems they seek to advance. iPSC systems are best viewed as being just another tool in the psychiatry researcher's armory; they have potentially unique utility for understanding fundamental cellular processes and simple network activity but are less suited for investigations of intact brain systems and, crucially, how complex brain functions changes across the dynamic neurodevelopmental landscape.

\section{How many lines are enough? Ensuring robustness and reproducibility}

Traditional cell culture experiments typically examine the effect of an experimental manipulation in a standard cell line (or lines), meaning only technical replicates - i.e. repeated measurements within the same sample - can be obtained. In contrast, iPSC experiments can investigate multiple sources of variation: technical (i.e. how consistent are the effects of individual experiments given the same starting iPSC-derived cells?), within-subjects (i.e, how consistent are results from different iPSC lines derived from the same donor?) and between-subjects (i.e. how consistent are findings across individuals?) The ability to study differences between groups, or the impact of a given manipulation on a range of different genetic backgrounds, has great potential for understanding the differences between individuals. However, it also raises important caveats regarding sample size and power (49).

In common with all between-subjects approaches, 'n' for the purposes of iPSC-based studies remains the number of lines obtained from different individuals, rather than different iPSC lines taken from a single donor or technical replicates. Given this constraint, studies that pool lines from multiple donors are becoming increasingly prominent (Figure 2) and permit cost-effective resolution of eQTLs $(50,51)$, as well as GWAS of cellular phenotypes (52). However, pooling is unsuitable for detecting donor-autonomous phenotypes and is currently only compatible with limited functional readouts (single cell RNASeq, whole genome sequencing or SNP array) as individual donors within cellular 'villages' must be disambiguated based on genomic information. Whilst technical advances aim to improve the scalability of single-donor iPSC studies (53), working with multiple lines simultaneously remains a significant logistical challenge due to the significant length of time needed per differentiation and the infrastructure (e.g. incubator space, personnel) required for larger-scale experiments. Therefore, large iPSC studies will likely involve staggered differentiations of different lines, which in turn necessitates the careful control of potential batch effects (54) and limits the number of distinct lines that can feasibly be included in a single experiment. Large-scale iPSC studies are beginning to emerge $(50,55,56)$, but even the biggest conducted to date use lines from fewer than 350 subjects. This poses significant challenges for case-control studies in psychiatry (and, indeed, for all complex disorders) where, at least at the level of the whole individual, biological effects of risk factors are small, meaning that large numbers of individuals are needed to detect them (49). It is plausible that group differences are more penetrant at the cellular level, since cellular phenotypes are 'closer' to the substantial genetic risk that underpins psychiatric disorders, but this remains to be proven. Furthermore, significant questions remain about the reproducibility of findings, since few studies include attempts at replication. Notable diversity remains in differentiation protocols and other methodological considerations across the field (57-60); in the absence of standardized protocols it is unclear how to interpret differences observed between methodologically distinct studies. 
In cases where a within-subject control can be used (e.g. an isogenic control, for genetic manipulations, or a vehicle control, for pharmacological interventions), it may be possible to draw reliable conclusions using only a few $(\sim 5-6)$ individual iPSC lines to ensure that findings are consistent across genetic backgrounds (49). In contrast, where the comparison of interest is between groups of subjects, it will typically be most appropriate to prioritize the study of iPSC lines obtained from different individuals over replicate lines from the same individual. Thus, the scale and complexity of an experiment will need to be guided by the key questions that it seeks to answer in order to deliver robust findings.

\section{Are 3D models the future?}

Psychiatric symptoms are associated with subtle and widespread changes in brain function; thus, the ideal model system would permit the study of relatively complex circuitry and interactions between regions. Organoids are therefore appealing as a relatively complex human neural system that are compatible with many existing technical approaches and can be readily manipulated. Many established protocols aim to produce cortical tissue $(57,61,62)$ including hippocampus, but models exist of other brain regions, including midbrain $(61,63)$ and cerebellum (64). Furthermore, a choroid plexus organoid has been recently developed, raising the exciting prospect of a novel in vitro model for testing for brain penetrance during drug development (65). Organoid models are not as widely used as 2D iPSC culture systems and are technically challenging to establish and analyze. However, there are several noteworthy limitations and unanswered questions with regards to current systems.

Early protocols relying on self-organization gave rise to organoids that showed substantial variability in their cellular composition, tissue identity and morphology (66). Technical refinements have improved reproducibility, although differences between batches and cell lines are still observed $(66,67)$. It will likely be possible to refine protocols further; however, for the time being, heterogeneity remains a significant issue and so it is crucial for experimenters to carefully design experiments to minimize its impact, for example by averaging across multiple batches and using single-cell RNA-Seq approaches to understand the cellular milieu. Organoids cultured for extended periods can contain diverse cell types, including astrocytes, dopamine neurons and multiple subtypes of forebrain neurons $(66,68)$. However, they do not given rise to the all major cell types found in human brain. For example, current organoids lack microglia and myelination (69), and many do not contain GABAergic interneurons (70), and so it is unclear how faithfully their neural circuitry mimics that of the intact brain. iPSC-derived neural, microglial and astrocytic precursors can integrate to form 3D cultures (71), allowing researchers to manipulate the cellular mix to fit their experimental needs. Nevertheless, the popular conceptualization of organoids as 'mini-brains' remains far from accurate: their organization is limited and often unpredictable, and their complexity and cellular diversity is a fraction of that seen in vivo.

Given their increasingly utility for measuring the formation of neural circuits, a crucial outstanding question is the extent to which brain organoids faithfully recapitulate neurodevelopment. Current findings are mixed. They contain a complex mix of neural cells and exhibit a quasi-laminar structure similar to superficial and deep layers of the cortex, and exhibit network activity $(68,72-74)$. The neurons within them can make long-range connections, both within the organoid and with neighboring structures in the culture system or in vivo in animals. However, a recent study suggested that brain organoid cells may show abnormal maturation, possibly as the result of cellular stress induced by the in vitro environment, and lack the fine spatial resolution seen in vivo (27). Thus, further studies are required to clarify which aspects of human neurodevelopment (if any) can be accurately modelled using organoids, emphasizing the need for caution when extrapolating from organoid findings to fundamental aspects of 
human neurobiology: it is certainly too early to conclude that findings from organoids provide proof for neurodevelopmental changes in vivo (11).

Researchers are using novel strategies to enrich for the cells types that are their focus (for example the recent development of oligodendrocyte spheroids (75)) and/or fusing different types of organoids into 'assembloids'. Some fusions are designed to study interactions between neurons with distinct regional identities; combining organoids with dorsal and ventral forebrain identities to study interneuron migration (76), for example, or fusing cortical organoids with thalamic or striatal organoids to study the formation and function of long-range axonal connections $(77,78)$. Brain organoids can also be fused with cell or tissue types that they lack (e.g. microglia or blood vessels) $(79,80)$. Impressively, a recent study demonstrated that distinct organoids with cortical, spinal and skeletal muscle identities can be successfully fused to form a functional cortical-motor circuit (81). Assembloids have yet to be widely used in psychiatry, but examples are beginning to emerge, such as the demonstration of altered migration in assembloids derived from patients with Timothy Syndrome (82). Ultimately, bioengineering approaches may allow the spatially-controlled delivery of external patterning cues, to further increase the sophistication and reproducibility of organoid and assembloid models $(79,83)$. However, even the most advanced models will likely never rival the complexity of the human brain; even if this were ever technically feasible it would raise substantial ethical questions (84).

\section{Can we unravel mechanisms of genetic risk using iPSCs?}

As the use of iPSC-based models in psychiatry has gained popularity, researchers have shifted their focus from patient vs. control studies to those seeking to understand the functional impact of disease-associated genetic risk. Rare and highly penetrant variants are particularly amenable to this approach as their large effect sizes (85) means that their impact can be studied betweensubjects in relatively small samples. Several groups have successfully used iPSC-based models to illuminate the impact of rare variants of relevance to psychiatry on neuronal function $(7,8)$, underscoring the utility of iPSC-based models for understanding highly penetrant variants. However, this approach is also amenable to investigation of the summative effects of common variants on cellular function, since extremes of polygene risk scores approach the odds ratios of many rare variants $(86)$.

Advances in genome engineering $(2,87-89)$ allow researchers to study the impact of genetic variants of interest, or to manipulate specific transcripts or epigenetic factors, on an isogenic background, thereby removing variance due to genetic differences between individuals (Figure 2; although the impact of variants should be studied on multiple lines to assess reproducibility and generalizability (49)). However, even with the use of isogenic lines, studying individual common variants using iPSC models is more challenging than for rare variants, as their effect sizes are far smaller (86). Moreover, it is typically unclear whether the SNPs identified by GWAS are directly relevant for pathophysiology, or whether they are simply in linkage disequilibrium with causal variants. Therefore, although appealing in theory, studies aiming to systematically (but agnostically) introduce GWAS risk associated alleles into iPSCs for functional screening are likely to be of limited use. Indeed, since most risk SNPs are non-coding, it is often not clear which the relevant gene or genes are, let alone how their function is altered in association with disease risk. We therefore suggest that researchers seeking to use iPSC models to investigate the biological impact of risk SNPs draw on information from the increasing number of largescale studies investigating gene expression and function in native human cells and tissues, and the ways in which these factors are affected by genetic variation $(44-46,48)$. Information of this type can be used not only to help to identify the specific genes tagged by individual risk SNPS (90), but also to clarify the pathophysiological mechanisms at a molecular level. It may then be more appropriate to model the molecular consequences of genetic variation, rather than directly 
recapitulating the genetic variant itself: a strategy that may be particularly useful in cases where the functional variant is unclear. For example, data obtained in postmortem human brain identified a small subset of schizophrenia risk loci that showed single gene expression quantitative trait loci (eQTLs); for one of these - rs4702 (86), in the furin gene - the schizophrenia-associated SNP is putatively the causal variant (91). Based on this information, Schrode and colleagues (6)modelled these single gene eQTLs in iPSC-derived neurons. Strikingly, as well as individually influencing aspects of synapse development and neuronal activity, the combination of these distinct perturbations altered the expression of genes enriched for those differentially expressed in psychiatric disorders (6). These findings highlight the potential of using information from human samples to refine iPSC models in which to explore pathophysiological mechanisms.

iPSC systems might be anticipated to be better powered to detect associations between risk SNPs and aspects of gene expression than postmortem studies, since a comparison of lines carrying risk SNPs vs. non-risk isogenic controls should eliminate noise arising from genetic background and, potentially, from environmental and perimortem factors (49). However, even where the causal SNP is known, there are several other factors that may undercut the assumption that gene expression phenotypes will necessarily be 'cleaner' in iPSCs than in human tissue. Some of these factors are technical in nature: for example, although iPSCs can be differentiated into a wide array of distinct cellular populations, they are not yet able to capture the full diversity of neural cell types, meaning that cell-type specific expression phenotypes (92) may be missed (although note that studies of bulk postmortem tissue can also fail to detect effects of SNPs on cell- and/or isoform-specific aspects of gene expression $(93,94)$ ). Other factors result directly from the ex vivo nature of the iPSC approach: specifically, it is possible that environmental factors may be required for certain risk SNPs to mediate their pathogenic effects (95), and therefore that their effects may not be observed in the absence of these environmental factors. Lastly, eQTLs can vary across cellular differentiation (96) and neurodevelopment (97), and across brain regions and cell types (94), meaning that a causative risk associated SNP may only have pathogenic implications in a particular cell population at a particular time of life. Thus, the relative merits of iPSC systems vs. postmortem studies for identifying eQTLs remains to be determined. Given the diversity and complexity of relationships between individual SNPs and different facets of gene expression, it seems unlikely that either approach will be 'superior' in all cases.

\section{Conclusions}

iPSCs offer access to live human brain cells, the opportunity to study cause and effect relationships, and to investigate how these differ between individuals. Their compatibility with a wide range of technical approaches and their ready manipulation by genome engineering makes them, in many ways, the ultimate tissue culture system. They are particularly well-suited to understanding how fundamental processes occurring in (and between) neural cells are affected by penetrant genetic risk factors, or by pharmacological and environmental manipulations. However, they are arguably less well-suited to the study of group differences between psychiatry patients and controls, both because of the large numbers likely needed to achieve statistical power and due to the challenges of determining which cellular phenotypes are meaningful. Even if statistically robust phenotypes associated with psychiatric illnesses and/or genetic risk for them are identified, it will be a challenging endeavor to demonstrate their validity and relevance in vivo. This problem is particularly acute in the case of iPSC phenotypes that map onto early human neurodevelopment, since there are currently no means to determine ground truth. 
Many of the challenges currently faced by iPSC approaches are not unique: researchers working with postmortem brain tissue and with conventional cell lines have been grappling with similar questions for many years $(98,99)$. The impact of iPSC-based findings will undoubtedly be maximized by seeking convergence with those acquired using other approaches that provide insight into cellular and network phenomena, be they obtained using traditional cell culture, or research in human samples or animal models (Figure 1, Table 1). The field will be best served by researchers remaining focused on their hypothesis of interest and clear-eyed about the suitability of iPSC-based models for testing it.

\section{Acknowledgements}

This study was supported by the National Institute for Health Research Oxford Health Biomedical Research Centre. The views expressed are those of the authors and not necessarily those of the NHS, the NIHR or the Department of Health. We are grateful for the vision and generosity of the Lieber and Maltz families, who made this work possible.

\section{Disclosures}

All authors report no biomedical financial interests or potential conflicts of interest with this work.

\section{References}

1. Sonntag K-C, Song B, Lee N, Jung JH, Cha Y, Leblanc P, et al. (2018): Pluripotent stem cell-based therapy for Parkinson's disease: Current status and future prospects. Progress in Neurobiology. 168:1-20.

2. Fernando MB, Ahfeldt T, Brennand KJ (2020): Modeling the complex genetic architectures of brain disease. Nature Genetics. 52:363-369.

3. Urbach A, Bar-Nur O, Daley GQ, Benvenisty N (2010): Differential Modeling of Fragile X Syndrome by Human Embryonic Stem Cells and Induced Pluripotent Stem Cells. Cell Stem Cell. 6:407-411.

4. Brennand KJ, Simone A, Jou J, Gelboin-Burkhart C, Tran N, Sangar S, et al. (2011): Modelling schizophrenia using human induced pluripotent stem cells. Nature. 473:221-225.

5. $\quad$ Mertens J, Wang Q-W, Kim Y, Yu DX, Pham S, Yang B, et al. (2015): Differential responses to lithium in hyperexcitable neurons from patients with bipolar disorder. Nature. 527:95-99.

6. $\quad$ Schrode N, Ho S-M, Yamamuro K, Dobbyn A, Huckins L, Matos MR, et al. (2019): Synergistic effects of common schizophrenia risk variants. Nature Genetics. 51:1475-1485. 7. Flaherty E, Zhu S, Barretto N, Cheng E, Deans PJM, Fernando MB, et al. (2019): Neuronal impact of patient-specific aberrant NRXN1 $\alpha$ splicing. Nature Genetics. 51:1679-1690. 8. Tian Y, Voineagu I, Paşca SP, Won H, Chandran V, Horvath S, et al. (2014): Alteration in basal and depolarization induced transcriptional network in iPSC derived neurons from Timothy syndrome. Genome Medicine. 6:75.

9. Li J, Ryan SK, Deboer E, Cook K, Fitzgerald S, Lachman HM, et al. (2019): Mitochondrial deficits in human iPSC-derived neurons from patients with 22q11.2 deletion syndrome and schizophrenia. Translational Psychiatry. 9:302.

10. Mariani J, Coppola G, Zhang P, Abyzov A, Provini L, Tomasini L, et al. (2015): FOXG1Dependent Dysregulation of GABA/Glutamate Neuron Differentiation in Autism Spectrum Disorders. Cell. 162:375-390.

11. Stachowiak EK, Benson CA, Narla ST, Dimitri A, Chuye LEB, Dhiman S, et al. (2017): Cerebral organoids reveal early cortical maldevelopment in schizophrenia-computational anatomy and genomics, role of FGFR1. Translational Psychiatry. 7:6. 
12. Kathuria A, Lopez-Lengowski K, Vater M, McPhie D, Cohen BM, Karmacharya R (2020): Transcriptome analysis and functional characterization of cerebral organoids in bipolar disorder. Genome Medicine. 12:34.

13. Mellios N, Feldman DA, Sheridan SD, Ip JPK, Kwok S, Amoah SK, et al. (2018): MeCP2-regulated miRNAs control early human neurogenesis through differential effects on ERK and AKT signaling. Molecular Psychiatry. 23:1051-1065.

14. Wang P, Mokhtari R, Pedrosa E, Kirschenbaum M, Bayrak C, Zheng D, et al. (2017): CRISPR/Cas9-mediated heterozygous knockout of the autism gene CHD8 and characterization of its transcriptional networks in cerebral organoids derived from iPS cells. Molecular Autism. 8:11.

15. Paşca SP, Portmann T, Voineagu I, Yazawa M, Shcheglovitov A, Paşca AM, et al. (2011): Using iPSC-derived neurons to uncover cellular phenotypes associated with Timothy syndrome. Nature Medicine. 17:1657-1662.

16. Clark MB, Wrzesinski T, Garcia AB, Hall NAL, Kleinman JE, Hyde T, et al. (2020): Longread sequencing reveals the complex splicing profile of the psychiatric risk gene CACNA1C in human brain. Molecular Psychiatry. 25:37-47.

17. Law AJ, Kleinman JE, Weinberger DR, Weickert CS (2007): Disease-associated intronic variants in the ErbB4 gene are related to altered ErbB4 splice-variant expression in the brain in schizophrenia. Human Molecular Genetics. 16:129-141.

18. Shao Z, Noh H, Bin Kim W, Ni P, Nguyen C, Cote SE, et al. (2019): Dysregulated protocadherin-pathway activity as an intrinsic defect in induced pluripotent stem cell-derived cortical interneurons from subjects with schizophrenia. Nature Neuroscience. 22:229-242. 19. Wang X, Christian KM, Song H, Ming G-I (2018): Synaptic dysfunction in complex psychiatric disorders: from genetics to mechanisms. Genome Medicine. 10:9.

20. Onwordi EC, Halff EF, Whitehurst T, Mansur A, Cotel M-C, Wells L, et al. (2020): Synaptic density marker SV2A is reduced in schizophrenia patients and unaffected by antipsychotics in rats. Nature Communications. 11:246.

21. Harrison PJ (1999): The neuropathology of schizophrenia: A critical review of the data and their interpretation. Brain. 122:593-624.

22. Madison JM, Zhou F, Nigam A, Hussain A, Barker DD, Nehme R, et al. (2015):

Characterization of bipolar disorder patient-specific induced pluripotent stem cells from a family reveals neurodevelopmental and mRNA expression abnormalities. Molecular Psychiatry. 20:703-717.

23. Harrison PJ, Colbourne L, Harrison CH (2020): The neuropathology of bipolar disorder: systematic review and meta-analysis. Molecular Psychiatry. 25:1787-1808.

24. Christison GW, Casanova MF, Weinberger DR, Rawlings R, Kleinman JE (1989): A Quantitative Investigation of Hippocampal Pyramidal Cell Size, Shape, and Variability of Orientation in Schizophrenia. Archives of General Psychiatry. 46:1027-1032.

25. Ruediger T, Bolz J (2007): Neurotransmitters and the Development of Neuronal Circuits. In: Bagnard D, editor. Axon Growth and Guidance. New York, NY: Springer New York, pp 104114.

26. Paolicelli RC, Bolasco G, Pagani F, Maggi L, Scianni M, Panzanelli P, et al. (2011): Synaptic Pruning by Microglia Is Necessary for Normal Brain Development. Science. 333:1456.

27. Bhaduri A, Andrews MG, Mancia Leon W, Jung D, Shin D, Allen D, et al. (2020): Cell stress in cortical organoids impairs molecular subtype specification. Nature. 578:142-148.

28. Hook V, Brennand Kristen J, Kim Y, Toneff T, Funkelstein L, Lee Kelly C, et al. (2014): Human iPSC Neurons Display Activity-Dependent Neurotransmitter Secretion: Aberrant Catecholamine Levels in Schizophrenia Neurons. Stem Cell Reports. 3:531-538.

29. Vadodaria KC, Jones JR, Linker S, Gage FH (2019): Modeling Brain Disorders Using Induced Pluripotent Stem Cells. Cold Spring Harbor Perspectives in Biology. 
30. Howes O, Bose S, Turkheimer F, Valli I, Egerton A, Stahl D, et al. (2011): Progressive increase in striatal dopamine synthesis capacity as patients develop psychosis: a PET study. Molecular Psychiatry. 16:885-886.

31. Laruelle M, Abi-Dargham A, Gil R, Kegeles L, Innis R (1999): Increased dopamine transmission in schizophrenia: relationship to illness phases. Biological Psychiatry. 46:56-72.

32. Kim K, Doi A, Wen B, Ng K, Zhao R, Cahan P, et al. (2010): Epigenetic memory in induced pluripotent stem cells. Nature. 467:285-290.

33. Polo JM, Liu S, Figueroa ME, Kulalert W, Eminli S, Tan KY, et al. (2010): Cell type of origin influences the molecular and functional properties of mouse induced pluripotent stem cells. Nature Biotechnology. 28:848-855.

34. Livesey MR, Magnani D, Hardingham GE, Chandran S, Wyllie DJA (2016): Functional properties of in vitro excitatory cortical neurons derived from human pluripotent stem cells. The Journal of Physiology. 594:6573-6582.

35. Gunhanlar N, Shpak G, van der Kroeg M, Gouty-Colomer LA, Munshi ST, Lendemeijer B, et al. (2018): A simplified protocol for differentiation of electrophysiologically mature neuronal networks from human induced pluripotent stem cells. Molecular Psychiatry. 23:1336-1344.

36. Burke EE, Chenoweth JG, Shin JH, Collado-Torres L, Kim S-K, Micali N, et al. (2020): Dissecting transcriptomic signatures of neuronal differentiation and maturation using iPSCs. Nature Communications. 11:462.

37. Weinberger DR (2017): Future of Days Past: Neurodevelopment and Schizophrenia. Schizophrenia Bulletin. 43:1164-1168.

38. Xiao Z, Qiu T, Ke X, Xiao X, Xiao T, Liang F, et al. (2014): Autism Spectrum Disorder as Early Neurodevelopmental Disorder: Evidence from the Brain Imaging Abnormalities in 23 Years Old Toddlers. Journal of Autism and Developmental Disorders. 44:1633-1640.

39. Moffat JG, Vincent F, Lee JA, Eder J, Prunotto M (2017): Opportunities and challenges in phenotypic drug discovery: an industry perspective. Nature Reviews Drug Discovery. 16:531543.

40. Marchetto MC, Belinson H, Tian Y, Freitas BC, Fu C, Vadodaria KC, et al. (2017): Altered proliferation and networks in neural cells derived from idiopathic autistic individuals. Molecular Psychiatry. 22:820-835.

41. Stern S, Santos R, Marchetto MC, Mendes APD, Rouleau GA, Biesmans S, et al. (2018): Neurons derived from patients with bipolar disorder divide into intrinsically different subpopulations of neurons, predicting the patients' responsiveness to lithium. Molecular Psychiatry. 23:1453-1465.

42. Stern S, Sarkar A, Galor D, Stern T, Mei A, Stern Y, et al. (2020): A Physiological Instability Displayed in Hippocampal Neurons Derived From Lithium-Nonresponsive Bipolar Disorder Patients. Biological Psychiatry. 88:150-158.

43. Stern S, Sarkar A, Stern T, Mei A, Mendes APD, Stern Y, et al. (2020): Mechanisms Underlying the Hyperexcitability of CA3 and Dentate Gyrus Hippocampal Neurons Derived From Patients With Bipolar Disorder. Biological Psychiatry. 88:139-149.

44. The GTEx Consortium (2020): The GTEx Consortium atlas of genetic regulatory effects across human tissues. Science. 369:1318.

45. Schubert Christian R, O'Donnell P, Quan J, Wendland Jens R, Xi Hualin S, Winslow Ashley R, et al. (2015): BrainSeq: Neurogenomics to Drive Novel Target Discovery for Neuropsychiatric Disorders. Neuron. 88:1078-1083.

46. Davis CA, Hitz BC, Sloan CA, Chan ET, Davidson JM, Gabdank I, et al. (2018): The Encyclopedia of DNA elements (ENCODE): data portal update. Nucleic Acids Research. 46:D794-D801.

47. Streeter I, Harrison PW, Faulconbridge A, The HipSci Consortium, Flicek P, Parkinson $\mathrm{H}$, et al. (2017): The human-induced pluripotent stem cell initiative-data resources for cellular genetics. Nucleic Acids Research. 45:D691-D697. 
48. Hoffman GE, Bendl J, Voloudakis G, Montgomery KS, Sloofman L, Wang Y-C, et al. (2019): CommonMind Consortium provides transcriptomic and epigenomic data for Schizophrenia and Bipolar Disorder. Scientific Data. 6:180.

49. Hoffman GE, Schrode N, Flaherty E, Brennand KJ (2019): New considerations for hiPSC-based models of neuropsychiatric disorders. Molecular Psychiatry. 24:49-66.

50. Cuomo ASE, Seaton DD, McCarthy DJ, Martinez I, Bonder MJ, Garcia-Bernardo J, et al. (2020): Single-cell RNA-sequencing of differentiating iPS cells reveals dynamic genetic effects on gene expression. Nature Communications. 11:810.

51. Schwartzentruber J, Foskolou S, Kilpinen H, Rodrigues J, Alasoo K, Knights AJ, et al. (2018): Molecular and functional variation in iPSC-derived sensory neurons. Nature Genetics. 50:54-61.

52. Mitchell JM, Nemesh J, Ghosh S, Handsaker RE, Mello CJ, Meyer D, et al. (2020):

Mapping genetic effects on cellular phenotypes with "cell villages". bioRxiv.2020.2006.2029.174383.

53. D'Aiuto L, Zhi Y, Kumar Das D, Wilcox MR, Johnson JW, McClain L, et al. (2014): Largescale generation of human iPSC-derived neural stem cells/early neural progenitor cells and their neuronal differentiation. Organogenesis. 10:365-377.

54. Tung P-Y, Blischak JD, Hsiao CJ, Knowles DA, Burnett JE, Pritchard JK, et al. (2017): Batch effects and the effective design of single-cell gene expression studies. Scientific Reports. 7:39921.

55. Kilpinen H, Goncalves A, Leha A, Afzal V, Alasoo K, Ashford S, et al. (2017): Common genetic variation drives molecular heterogeneity in human iPSCs. Nature. 546:370-375.

56. DeBoever C, Li H, Jakubosky D, Benaglio P, Reyna J, Olson KM, et al. (2017): Large-

Scale Profiling Reveals the Influence of Genetic Variation on Gene Expression in Human Induced Pluripotent Stem Cells. Cell Stem Cell. 20:533-546.e537.

57. Paşca AM, Sloan SA, Clarke LE, Tian Y, Makinson CD, Huber N, et al. (2015):

Functional cortical neurons and astrocytes from human pluripotent stem cells in 3D culture. Nature Methods. 12:671-678.

58. Chambers SM, Fasano CA, Papapetrou EP, Tomishima M, Sadelain M, Studer L (2009): Highly efficient neural conversion of human ES and iPS cells by dual inhibition of SMAD signaling. Nature Biotechnology. 27:275-280.

59. Shi Y, Kirwan P, Livesey FJ (2012): Directed differentiation of human pluripotent stem cells to cerebral cortex neurons and neural networks. Nature Protocols. 7:1836-1846.

60. Zhang Y, Pak C, Han Y, Ahlenius H, Zhang Z, Chanda S, et al. (2013): Rapid SingleStep Induction of Functional Neurons from Human Pluripotent Stem Cells. Neuron. 78:785-798. 61. Qian X, Nguyen Ha N, Song Mingxi M, Hadiono C, Ogden Sarah C, Hammack C, et al. (2016): Brain-Region-Specific Organoids Using Mini-bioreactors for Modeling ZIKV Exposure. Cell. 165:1238-1254.

62. Mariani J, Simonini MV, Palejev D, Tomasini L, Coppola G, Szekely AM, et al. (2012): Modeling human cortical development in vitro using induced pluripotent stem cells. Proceedings of the National Academy of Sciences. 109:12770.

63. Jo J, Xiao Y, Sun Alfred X, Cukuroglu E, Tran H-D, Göke J, et al. (2016): Midbrain-like Organoids from Human Pluripotent Stem Cells Contain Functional Dopaminergic and Neuromelanin-Producing Neurons. Cell Stem Cell. 19:248-257.

64. Muguruma K, Nishiyama A, Kawakami H, Hashimoto K, Sasai Y (2015): SelfOrganization of Polarized Cerebellar Tissue in 3D Culture of Human Pluripotent Stem Cells. Cell Reports. 10:537-550.

65. Pellegrini L, Bonfio C, Chadwick J, Begum F, Skehel M, Lancaster MA (2020): Human CNS barrier-forming organoids with cerebrospinal fluid production. Science. 369:eaaz5626. 
66. Quadrato G, Nguyen T, Macosko EZ, Sherwood JL, Min Yang S, Berger DR, et al. (2017): Cell diversity and network dynamics in photosensitive human brain organoids. Nature. 545:48-53.

67. Lancaster MA, Corsini NS, Wolfinger S, Gustafson EH, Phillips AW, Burkard TR, et al. (2017): Guided self-organization and cortical plate formation in human brain organoids. Nature Biotechnology. 35:659-666.

68. Velasco S, Kedaigle AJ, Simmons SK, Nash A, Rocha M, Quadrato G, et al. (2019): Individual brain organoids reproducibly form cell diversity of the human cerebral cortex. Nature. 570:523-527.

69. Heide M, Huttner WB, Mora-Bermúdez F (2018): Brain organoids as models to study human neocortex development and evolution. Current Opinion in Cell Biology. 55:8-16.

70. Bagley JA, Reumann D, Bian S, Lévi-Strauss J, Knoblich JA (2017): Fused cerebral organoids model interactions between brain regions. Nature methods. 14:743-751.

71. Park J, Wetzel I, Marriott I, Dréau D, D’Avanzo C, Kim DY, et al. (2018): A 3D human triculture system modeling neurodegeneration and neuroinflammation in Alzheimer's disease. Nature Neuroscience. 21:941-951.

72. Qian X, Su Y, Adam CD, Deutschmann AU, Pather SR, Goldberg EM, et al. (2020): Sliced Human Cortical Organoids for Modeling Distinct Cortical Layer Formation. Cell Stem Cell. 26:766-781.e769.

73. Giandomenico SL, Mierau SB, Gibbons GM, Wenger LMD, Masullo L, Sit T, et al. (2019): Cerebral organoids at the air-liquid interface generate diverse nerve tracts with functional output. Nature Neuroscience. 22:669-679.

74. Amiri A, Coppola G, Scuderi S, Wu F, Roychowdhury T, Liu F, et al. (2018):

Transcriptome and epigenome landscape of human cortical development modeled in organoids. Science. 362:eaat6720.

75. Marton RM, Miura Y, Sloan SA, Li Q, Revah O, Levy RJ, et al. (2019): Differentiation and maturation of oligodendrocytes in human three-dimensional neural cultures. Nature Neuroscience. 22:484-491.

76. Xiang Y, Tanaka Y, Patterson B, Kang Y-J, Govindaiah G, Roselaar N, et al. (2017): Fusion of Regionally Specified hPSC-Derived Organoids Models Human Brain Development and Interneuron Migration. Cell Stem Cell. 21:383-398.e387.

77. Xiang Y, Tanaka Y, Cakir B, Patterson B, Kim K-Y, Sun P, et al. (2019): hESC-Derived Thalamic Organoids Form Reciprocal Projections When Fused with Cortical Organoids. Cell Stem Cell. 24:487-497.e487.

78. Miura Y, Li M-Y, Birey F, Ikeda K, Revah O, Thete MV, et al. (2020): Generation of human striatal organoids and cortico-striatal assembloids from human pluripotent stem cells. Nature Biotechnology. 38:1421-1430.

79. Marton RM, Pașca SP (2020): Organoid and Assembloid Technologies for Investigating Cellular Crosstalk in Human Brain Development and Disease. Trends in Cell Biology. 30:133143.

80. Xiang Y, Cakir B, Park I-H (2020): Deconstructing and reconstructing the human brain with regionally specified brain organoids. Seminars in Cell \& Developmental Biology.

81. Andersen J, Revah O, Miura Y, Thom N, Amin ND, Kelley KW, et al. (2020): Generation of Functional Human 3D Cortico-Motor Assembloids. Cell. 183:1913-1929.e1926.

82. Birey F, Andersen J, Makinson CD, Islam S, Wei W, Huber N, et al. (2017): Assembly of functionally integrated human forebrain spheroids. Nature. 545:54-59.

83. Yin X, Mead Benjamin E, Safaee H, Langer R, Karp Jeffrey M, Levy O (2016):

Engineering Stem Cell Organoids. Cell Stem Cell. 18:25-38.

84. Lavazza A, Massimini M (2018): Cerebral organoids: ethical issues and consciousness assessment. Journal of Medical Ethics. 44:606. 
85. Giegling I, Hosak L, Mössner R, Serretti A, Bellivier F, Claes S, et al. (2017): Genetics of schizophrenia: A consensus paper of the WFSBP Task Force on Genetics. The World Journal of Biological Psychiatry. 18:492-505.

86. Ripke S, Neale BM, Corvin A, Walters JTR, Farh K-H, Holmans PA, et al. (2014): Biological insights from 108 schizophrenia-associated genetic loci. Nature. 511:421-427.

87. Matos MR, Ho S-M, Schrode N, Brennand KJ (2020): Integration of CRISPRengineering and hiPSC-based models of psychiatric genomics. Molecular and Cellular Neuroscience. 107:103532.

88. Carroll D (2011): Genome Engineering With Zinc-Finger Nucleases. Genetics. 188:773.

89. Joung JK, Sander JD (2013): TALENs: a widely applicable technology for targeted genome editing. Nature Reviews Molecular Cell Biology. 14:49-55.

90. Gusev A, Mancuso N, Won H, Kousi M, Finucane HK, Reshef Y, et al. (2018): Transcriptome-wide association study of schizophrenia and chromatin activity yields mechanistic disease insights. Nature Genetics. 50:538-548.

91. Fromer M, Roussos P, Sieberts SK, Johnson JS, Kavanagh DH, Perumal TM, et al. (2016): Gene expression elucidates functional impact of polygenic risk for schizophrenia. Nature Neuroscience. 19:1442-1453.

92. Donovan MKR, D'Antonio-Chronowska A, D'Antonio M, Frazer KA (2020): Cellular deconvolution of GTEx tissues powers discovery of disease and cell-type associated regulatory variants. Nature Communications. 11:955.

93. Yao DW, O'Connor LJ, Price AL, Gusev A (2020): Quantifying genetic effects on disease mediated by assayed gene expression levels. Nature Genetics. 52:626-633.

94. Jaffe AE, Hoeppner DJ, Saito T, Blanpain L, Ukaigwe J, Burke EE, et al. (2020): Profiling gene expression in the human dentate gyrus granule cell layer reveals insights into schizophrenia and its genetic risk. Nature Neuroscience. 23:510-519.

95. Ursini G, Punzi G, Chen Q, Marenco S, Robinson JF, Porcelli A, et al. (2018):

Convergence of placenta biology and genetic risk for schizophrenia. Nature Medicine. 24:792-

801.

96. Strober BJ, Elorbany R, Rhodes K, Krishnan N, Tayeb K, Battle A, et al. (2019): Dynamic genetic regulation of gene expression during cellular differentiation. Science. 364:1287.

97. O'Brien HE, Hannon E, Hill MJ, Toste CC, Robertson MJ, Morgan JE, et al. (2018): Expression quantitative trait loci in the developing human brain and their enrichment in neuropsychiatric disorders. Genome Biology. 19:194.

98. Lewis DA (2002): The Human Brain Revisited: Opportunities and Challenges in Postmortem Studies of Psychiatric Disorders. Neuropsychopharmacology. 26:143-154.

99. Kleinman JE, Law AJ, Lipska BK, Hyde TM, Ellis JK, Harrison PJ, et al. (2011): Genetic Neuropathology of Schizophrenia: New Approaches to an Old Question and New Uses for Postmortem Human Brains. Biological Psychiatry. 69:140-145.

100. Krishna A, Biryukov M, Trefois C, Antony PMA, Hussong R, Lin J, et al. (2014): Systems genomics evaluation of the SH-SY5Y neuroblastoma cell line as a model for Parkinson's disease. BMC Genomics. 15:1154-1154.

101. Sridharan B, Hubbs C, Llamosas N, Kilinc M, Singhera FU, Willems E, et al. (2019): A Simple Procedure for Creating Scalable Phenotypic Screening Assays in Human Neurons. Scientific Reports. 9:9000.

102. Townsley KG, Brennand KJ, Huckins LM (2020): Massively parallel techniques for cataloguing the regulome of the human brain. Nature Neuroscience. 23:1509-1521.

103. Kampmann M (2020): CRISPR-based functional genomics for neurological disease. Nature Reviews Neurology. 16:465-480. 
104. Tian R, Gachechiladze MA, Ludwig CH, Laurie MT, Hong JY, Nathaniel D, et al. (2019): CRISPR Interference-Based Platform for Multimodal Genetic Screens in Human iPSC-Derived Neurons. Neuron. 104:239-255.e212.

105. Esk C, Lindenhofer D, Haendeler S, Wester RA, Pflug F, Schroeder B, et al. (2020): A human tissue screen identifies a regulator of ER secretion as a brain-size determinant. Science. 370:935. 


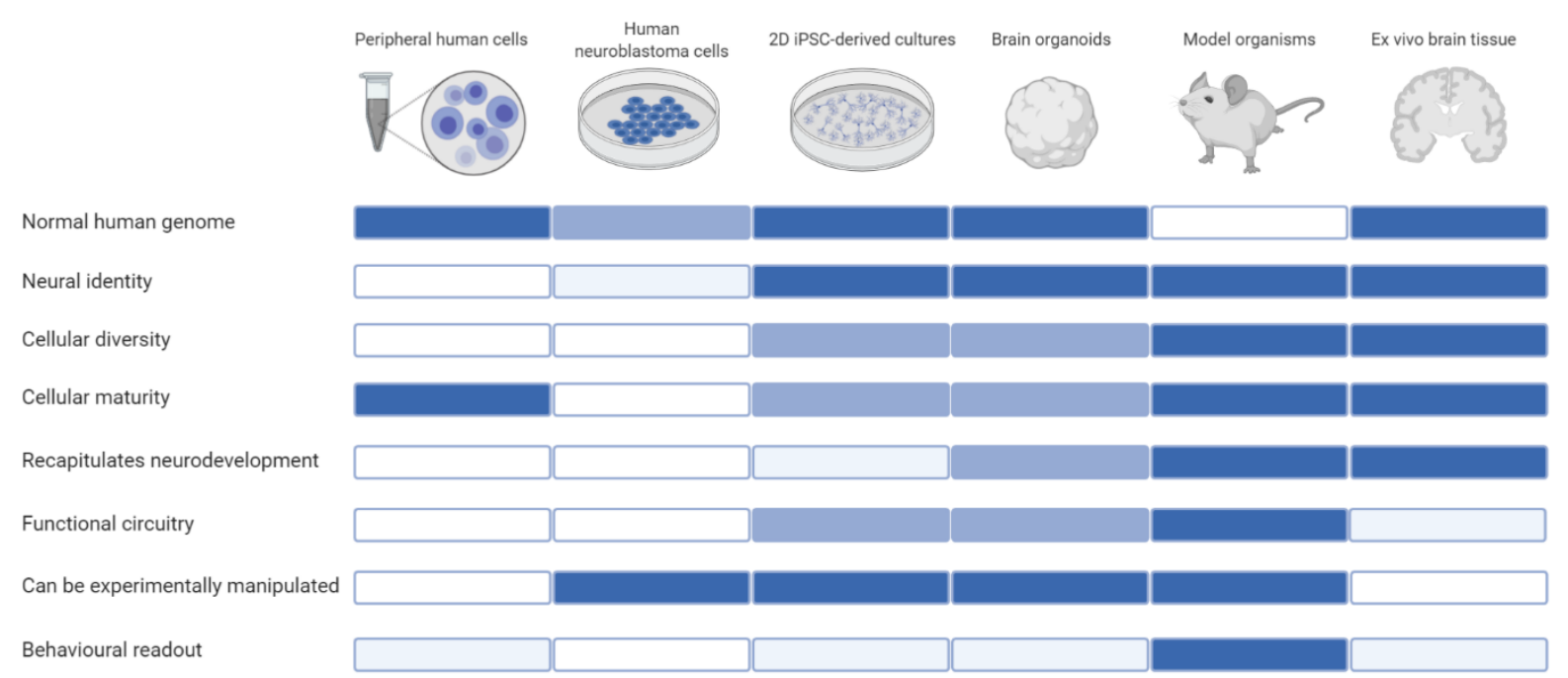

Figure 1. The strengths and limitations of different models of the intact human brain The strengths and limitations of six different model systems - human cells obtained peripherally from living donors (or immortalised versions of these cells, e.g. lymphoblast lines), neuroblastoma cells grown in standard cell culture, 2D and 3D iPSC models, animal models, and ex vivo human brain tissue (obtained either post-mortem or resected during surgery) - are presented. Each is rated for each of the factors listed left, with the intensity of the colour bar indicating the relative fidelity of each model. For example, cells and tissues derived from humans (including iPSCs) carry the genome of the donor, whilst the genome of human neuroblastoma lines contains various structural rearrangements and animal models carry their species' genome (100). In contrast, animal models are the only system in which behaviour can be dynamically linked to invasive measures of cellular and circuit function, although it is possible to correlate phenotypes observed in human cells and tissues with behavioural phenotypes observed in the donors from whom they were derived. The other factors are discussed in more detail in the main body of the review. Note that the colour bars are intended to give a broad, rather than definitive, indication of model fidelity: there is likely to be significant variation within categories, particularly in the case of iPSC models given the diversity of methodological approaches (e.g. co-culturing iPSC-derived neural progenitors with astrocytes accelerates maturation (36)). Created with BioRender.com. 


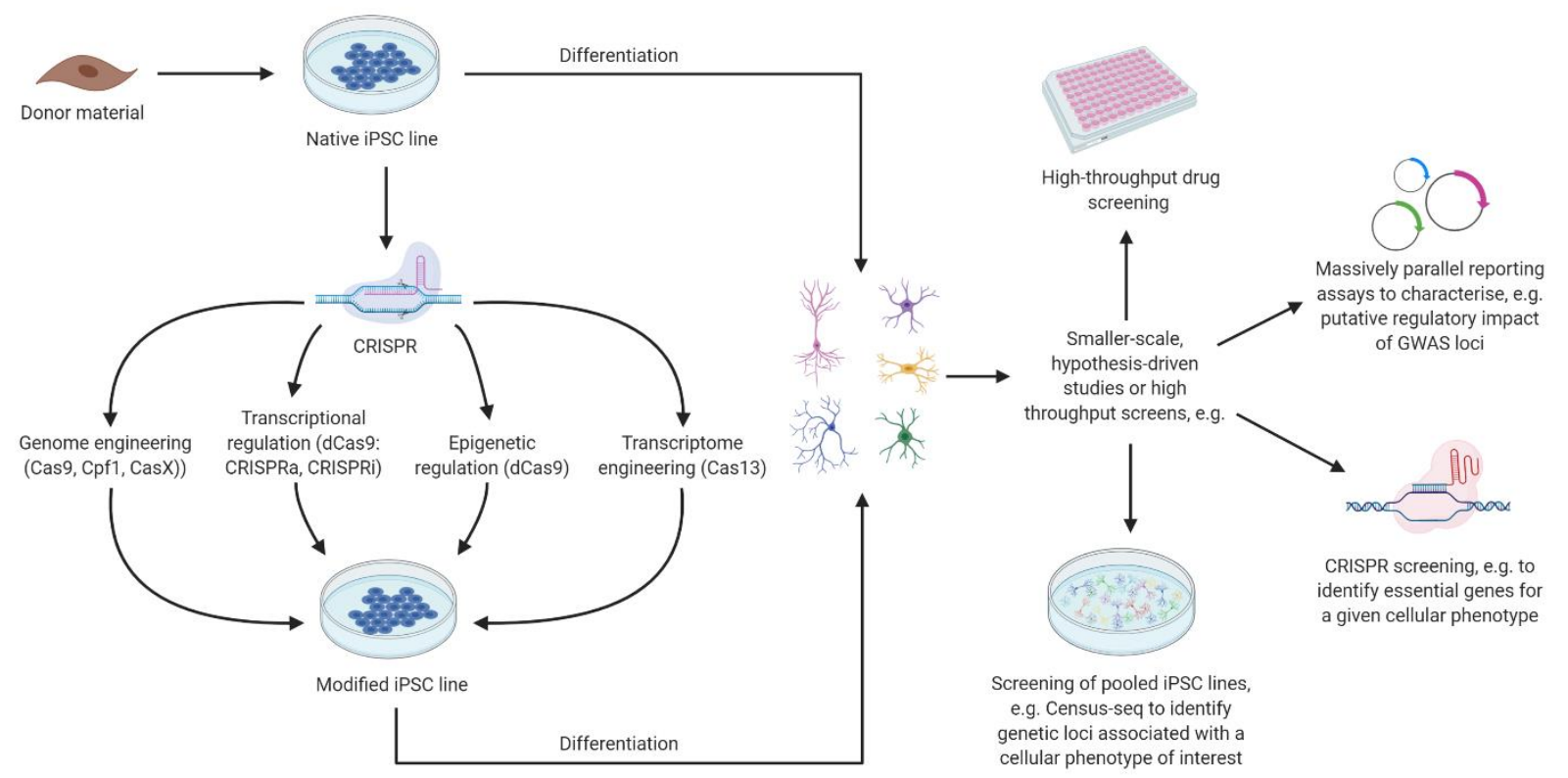

Figure 2. The diverse applications of human iPSC models

Rapid developments in genome engineering and iPSC-compatible systems means that human iPSC-derived models can be employed in a large number of ways in psychiatry. On the left of the figure is an overview of the many aspects of genomic and transcriptomic factors that can be modified using different forms of CRISPR (detailed in (2)). Both native and edited cells can then be used for a wide variety of applications; examples are shown on the right. (See (101) for drug screening, (102) for massively parallel reporter assays, $(103,104)$ for CRISPR screening; (52) for Census-seq). To date, most studies have employed 2D iPSC systems; however, many of these approaches, e.g. CRISPR screening (105), are also compatible with 3D systems. Created with BioRender.com 
Table 1: Recommendations for iPSC studies in psychiatry

\begin{tabular}{|c|c|}
\hline In experimental design & In publication \\
\hline $\begin{array}{l}\text { Consider whether an iPSC-based model is the } \\
\text { optimal system for the specific research question } \\
\text { (see Figure 1). Consider the following: } \\
\text { - } \quad \text { Are neural cells required? } \\
\text { - } \quad \text { Are human cells needed? } \\
\text { - } \quad \text { Are living cells/tissue required? } \\
\text { - } \quad \text { Can the question be answered in immature } \\
\text { cells/tissue? } \\
\text { - Does the question require access to intact, } \\
\text { complex neural circuitry? }\end{array}$ & $\begin{array}{l}\text { Ensure iPSC lines and methods are clearly and } \\
\text { fully described, including: } \\
\text { - The iPSC source (to allow readers to } \\
\text { ascertain overlap between studies) } \\
\text { - The number of donors included (and distinct } \\
\text { donor lines, where relevant) } \\
\text { - The number of technical replicates and } \\
\text { measures of their variability, including both } \\
\text { the number of differentiations, and the } \\
\text { number of wells/organoids), as appropriate } \\
\text { - The differentiation protocol }\end{array}$ \\
\hline $\begin{array}{l}\text { Determine the most appropriate iPSC system for the } \\
\text { experiment in question, bearing in mind: } \\
\text { - Selection of the type of cell, method of } \\
\text { generation, considerations of purity, timepoint, } \\
\text { inter- and intra-individual and -experiment } \\
\text { variation in vitro } \\
\text { - To what extent is the clinical trait heritable and } \\
\text { life-time stable? Do we know the causal cell } \\
\text { types underlying disease risk and progression? } \\
\text { - Whether or not the phenotype is cell- } \\
\text { autonomous (i.e. observed at the level of an } \\
\text { individual cell, without requirement for } \\
\text { interactions with other cells). } \\
\text { The technical approach required to assess the } \\
\text { phenotype (see Figure 1) } \\
\text { The level of cellular complexity and maturity } \\
\text { required }\end{array}$ & $\begin{array}{l}\text { Assess and report the cellular mix comprising the } \\
\text { iPSC model system, and how this varies between } \\
\text { technical replicates and donors }\end{array}$ \\
\hline $\begin{array}{l}\text { Identify the most meaningful phenotype (or range of } \\
\text { phenotypes), mindful that 'one size is unlikely to fit } \\
\text { all'. }\end{array}$ & $\begin{array}{l}\text { Perform and report power calculations for } \\
\text { between-subject analyses }\end{array}$ \\
\hline $\begin{array}{l}\text { Ensure that between-subject studies are } \\
\text { appropriately powered. We recommend for such } \\
\text { studies that researchers prioritise adding additional } \\
\text { donors over multiple lines from the same donors to } \\
\text { maximise statistical power (49) }\end{array}$ & $\begin{array}{l}\text { Where possible, in the case of gene expression } \\
\text { studies, attempt to validate iPSC-based findings } \\
\text { using publicly available gene expression data } \\
\text { from human post mortem brain, particularly that } \\
\text { obtained from foetal tissue. }\end{array}$ \\
\hline $\begin{array}{l}\text { Where isogenic controls are to be used, assess the } \\
\text { genetic manipulation of interest in lines from } \\
\text { multiple donors, to assess consistency across genetic } \\
\text { backgrounds ( } 49 \text { ) }\end{array}$ & $\begin{array}{l}\text { Conclusions should be reported with appropriate } \\
\text { caution and moderation, mindful of the } \\
\text { limitations of current iPSC systems, and the } \\
\text { challenges associated with validating their } \\
\text { findings in vivo. }\end{array}$ \\
\hline
\end{tabular}

These suggestions are not intended to be proscriptive, but to summarise and highlight to the key issues that merit discussion and consideration, and our approach to them. 